\title{
Endoscopic sinus surgery for the odontogenic maxillary cysts*
}

\author{
Satoshi Seno ${ }^{1}$, Takao Ogawa1, Masayuki Shibayama ${ }^{1}$, Fumio Ogawa ${ }^{1}$, Jun Fukui ${ }^{1}$, \\ Shigehiro Owaki ${ }^{1}$, Mikio Suzuki ${ }^{2}$, Takeshi Shimizu ${ }^{1}$ \\ Department of Otorhinolaryngology, Shiga University of Medical Science, Setatsukinowa, Otsu, Shiga, Japan \\ 2 Department of Otorhinolaryngology - Head and Neck Surgery, University of the Ryukyu, Naha, Okinawa, \\ Japan
}

\begin{abstract}
SUMMARY
Objective: To evaluate the effectiveness and usefulness of transnasal endoscopic surgery for the treatment of odontogenic maxillary cysts.

Methods: Between February 2003 and February 2008, transnasal endoscopic surgery was performed under general anesthesia in 13 patients (male 6 and female 7, 19 to 75 years old) with odontogenic maxillary cysts that extended to the maxillary sinus. Ten patients had a radicular cyst and three patients had a dentigerous cyst. After the resection of anterior edge of the inferior turbinate, the lateral wall of the inferior nasal meatus was opened. Then, the cyst wall of the maxillary sinus was partially or completely removed under the endoscope.

Results: The cyst walls were completely removed in five of ten patients with a radicular cyst and in all three patients with a dentigerous cyst. Five patients with a radicular cyst received partial resection of the cyst wall. The affected teeth could be preserved in seven of ten patients with a radicular cyst and in one of three patients with a dentigerous cyst. There were no complications, and postoperative courses were uneventful. Follow-up period ranged from 11 to 72 months (mean 42 months), and no recurrence has been noted in any of the cases.

Conclusions: Endoscopic transnasal surgery for the odontogenic maxillary cyst is less invasive than conventional dental approach, and most of the affected teeth can be preserved. This technique appears to be a simple and highly effective surgical treatment for the treatment of patients with odontogenic cysts that extend to the maxillary sinus.
\end{abstract}

Key words: endoscopic surgery, odontogenic cyst, radicular cyst, dentigerous cyst

\section{INTRODUCTION}

Endoscopic sinus surgery is widely used for the treatment of patients with chronic rhinosinusitis. Recently, the indications for endoscopic surgery have been extended to other nasal diseases, such as postoperative sinus cysts, benign neoplasms of the nasal cavity ${ }^{(1,2)}$, CSF fistulas ${ }^{(3)}$, blowout fractures of the orbital floor ${ }^{(4)}$, decompression of Grave's ophthalmopathy ${ }^{(5)}$, and ligation of the sphenopalatine and maxillary artery ${ }^{(6)}$.

The dental procedures have been used for the treatment of patients with odontogenic maxillary cysts; radicular cysts and dentigerous cysts. These require a gingival incision, and most of the affected teeth are extracted. Oroantral fistula and chronic rhinosinusitis are major complications associated with this procedure. However, when the cysts extend to the maxillary sinus, an endoscopic intranasal approach may be attempted, aiming to prevent any major complications.

In this article, we present our experience with intranasal endoscopic surgery for the treatment of radicular cysts and dentige- nous cysts involving the maxillary sinus. This technique is less invasive than traditional dental procedures, and most of the affected teeth can be preserved. The success rate, preservation rate of the affected teeth, and complications will be discussed.

\section{MATERIALS AND METHODS}

Subjects

Between February 2003 and February 2008, transnasal endoscopic surgery was performed in 13 patients with odontogenic maxillary cysts that extended to the maxillary sinus in our University Hospital. Ten patients had a radicular cyst (male 4, female 6; age range, 20 to 75 years) and three patients had a dentigerous cyst (male 2, female 1; age range, 19 to 60 years). Before the endoscopic surgery, it was decided that the undamaged teeth should be preserved in patients with a radicular cyst. In patients with a dentigerous cyst, the affected teeth would be preserved only if it was expected that they would grow in the correct position. 


\section{Operation}

All operations were performed under general anesthesia with controlled hypotension. The following endoscopic procedure was performed with a $4-\mathrm{mm}$ rigid endoscope $\left(0^{\circ}\right.$ and $\left.70^{\circ}\right)$. The anterior edge of the inferior turbinate was resected to visualize the lateral wall of the inferior meatus. A small mucoperiosteal flap from the lateral wall was made, and the bony wall of the inferior meatus was opened. The cyst wall was observed in the maxillary sinus, and it was partially or completely removed endoscopically from the maxillary sinus using forceps or curettes. After removing the cyst wall, the mucoperiosteal flap was layered on the floor of maxillary sinus.

\section{RESULTS}

Table 1 summarizes the patients and outcomes of the endoscopic surgery for the treatment of odontogenic maxillary cysts. The cyst walls were completely removed in five of ten patients with a radicular cyst and in all 3 patients with a dentigerous cyst. Five patients with a radicular cyst underwent partial resection of the cyst wall. The affected teeth could be preserved in seven of ten patients with a radicular cyst, and in one of three patients with a dentigerous cyst. The postoperative courses were uneventful. There were no complications such as oroantral fistula and chronic rhinosinusitis, following the surgery in this series. The follow-up period ranged from 11 to 72 months (mean 42 months), and no recurrence has been noted in any of the cases.

\section{CASE REPORT (Number 10)}

A 48-year-old woman was referred to the Department of Otorhinolaryngology, Shiga University hospital, complaining of right cheek pain and upper gingival swelling of three months' duration. Computed tomography (CT) showed a large cystic mass with a thin bony layer that extended into the right maxillary sinus (Figure 1). Dental examination revealed a radicular cyst arising from the right upper second molar. The affected tooth received the dental treatment prior to the endoscopic surgery. After the fenestration of the lateral wall of inferior
Table 1. Patients and outcome of the endoscopic transnasal surgery for odontogenic maxillary cysts. The post operative courses were uneventful and there were no complications during or after the surgery in this series. No recurrence has been noted after 11 to 72 months follow-up.

\begin{tabular}{|c|c|c|c|c|c|c|}
\hline \multicolumn{2}{|c|}{$\begin{array}{c}\text { No. Type of } \\
\text { cyst }\end{array}$} & $\begin{array}{l}\text { Age } \\
\text { (years) } \\
\text { /Gender }\end{array}$ & $\begin{array}{l}\text { Cyst wall } \\
\text { removal }\end{array}$ & $\begin{array}{l}\text { Affected } \\
\text { tooth } \\
\text { (months) }\end{array}$ & $\begin{array}{l}\text { Follow } \\
\text {-up } \\
\text { Duration }\end{array}$ & $\begin{array}{l}\text { Recur- } \\
\text { rence }\end{array}$ \\
\hline 1 & Radicular & 55/Male & Complete & Preserved & 72 & No \\
\hline 2 & Radicular & 61/Male & Partial & Extracted & 67 & No \\
\hline 3 & Radicular & 38/Male & Complete & Extracted & 57 & No \\
\hline 4 & Dentigerous & 27/Male & Complete & Extracted & 56 & No \\
\hline 5 & Dentigerous & 19/Male & Complete & Extracted & 54 & No \\
\hline 6 & Radicular & 75/Female & Complete & Preserved & 47 & No \\
\hline 7 & Dentigerous & 60/Female & Complete & Preserved & 37 & No \\
\hline 8 & Radicular & 42/Male & Partial & Preserved & 35 & No \\
\hline 9 & Radicular & 20/Female & Partial & Preserved & 34 & No \\
\hline 10 & Radicular & 48/Female & Complete & Preserved & 34 & No \\
\hline 11 & Radicular & 44/Female & Complete & Preserved & 30 & No \\
\hline 12 & Radicular & 28/Female & Partial & Extracted & 15 & No \\
\hline 13 & Radicular & 63/Female & Partial & Preserved & 11 & No \\
\hline
\end{tabular}

meatus, the cyst wall could be observed in the right maxillary sinus (Figure 2). On opening of the radicular cyst, a purulent discharge was noted (Figure 3), and the cyst wall was completely removed (Figure 4). The affected tooth was preserved, and a gingival incision was not needed. The patient's postoperative course was uneventful. The floor of the maxillary sinus was well epithelized at 3 months after the surgery (Figure 5). The complete removal of the radicular cyst was confirmed by the postoperative CT at 6 months after the surgery (Figure 6). No recurrence has been noted at more than 34 months of follow-up.

\section{DISCUSSION}

Odontogenic cysts are lesions that rise in the maxilla and/or mandible, originating from epithelial remains associated with odontogenesis. The most frequent type is a radicular cyst (65\%), followed by dentigerous cysts (24\%) and odontogenic
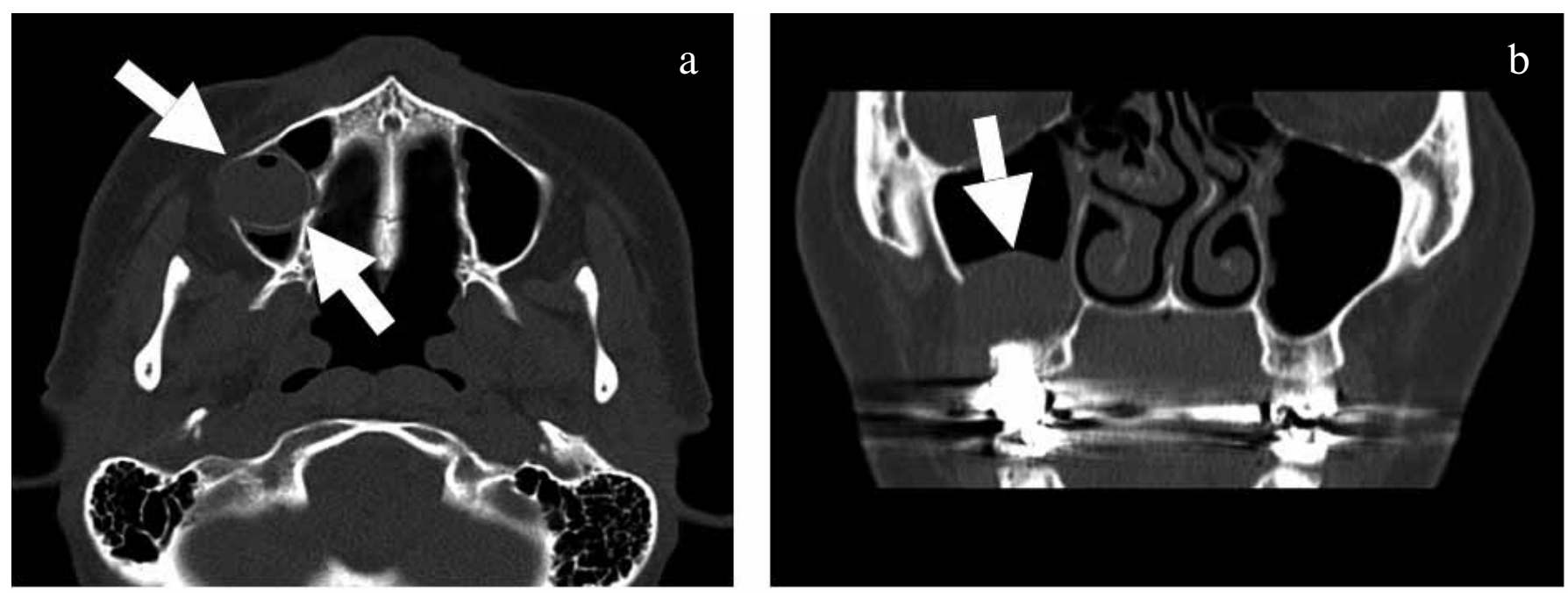

Figure 1. Axial (a) and coronal (b) CT scan showing a large cystic mass with a thin bony layer that extends into the right maxillary sinus (arrows). 


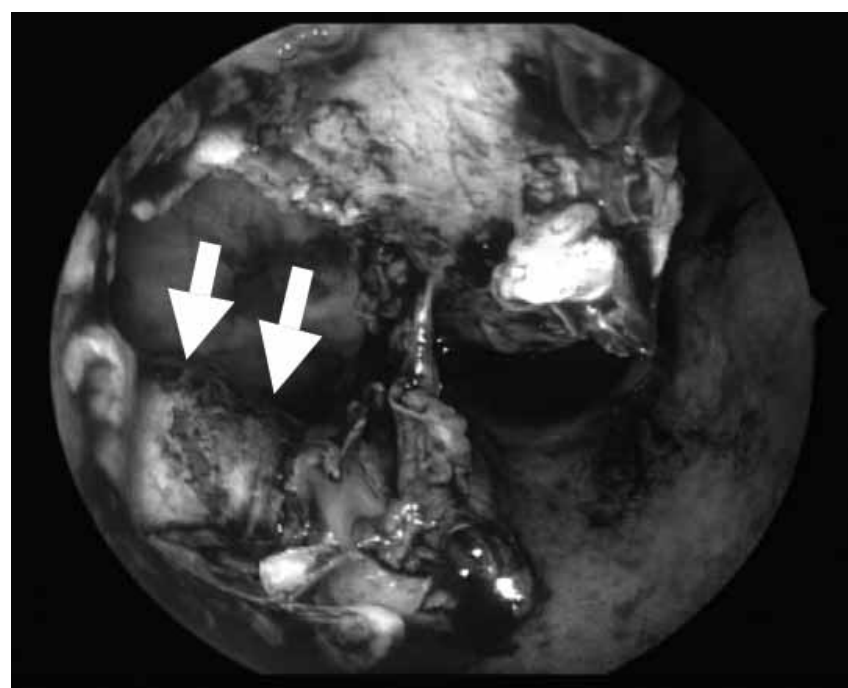

Figure 2. Endoscopic photograph showing the radicular cyst in the right maxillary sinus. After the fenestration of the lateral wall of inferior meatus, the cyst wall was observed in the maxillary sinus (arrows).

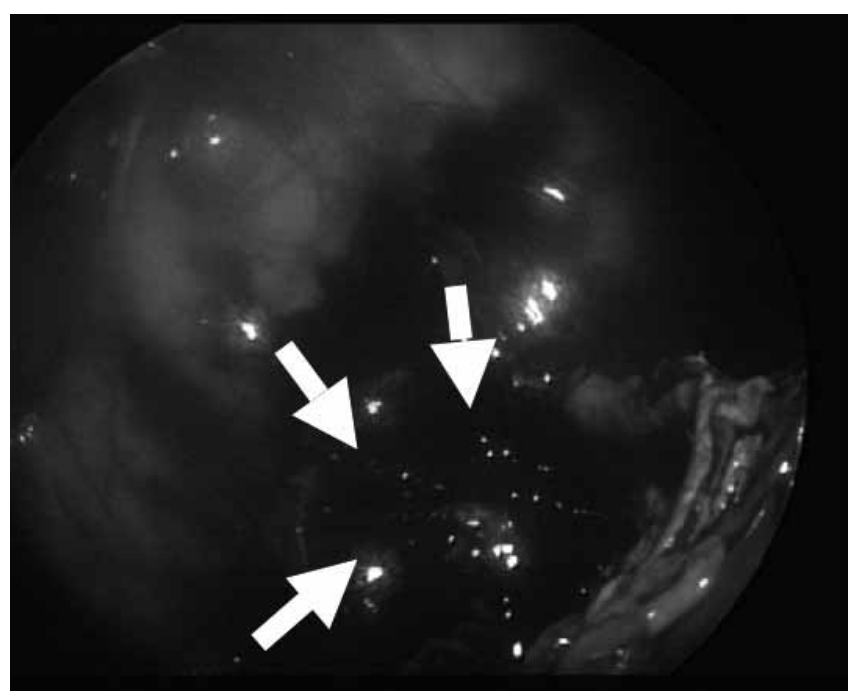

Figure 4. Endoscopic photograph showing the right maxillary sinus after the complete resection of the cyst wall of radicular cyst (arrows).

keratocysts $(5 \%)^{(7)}$. The majority of these cysts have been treated by dentists using surgical approaches such as enucleation, curettage, marsupialization, and tooth extraction ${ }^{(8)}$. However, most of the affected teeth were extracted along with the cyst walls. The complications, such as oroantral fistulas and chronic rhinosinusitis, may be caused by dental extractions in odontogenic cysts which involve the maxillary sinus.

In the last decade, endoscopic sinus surgery has been widely used, and the indications for endoscopic procedures were extended to many nasal diseases. For the treatment of patients with odontogenic maxillary cysts, endoscopic treatment has been used in addition to the dental procedure. SuarezConqueiro et al. ${ }^{(9)}$ reported the endoscopically assisted dental approach for the removal of an ectopic lower third molar tooth associated with a dentigerous cyst. Costa et al. ${ }^{(10)}$ performed

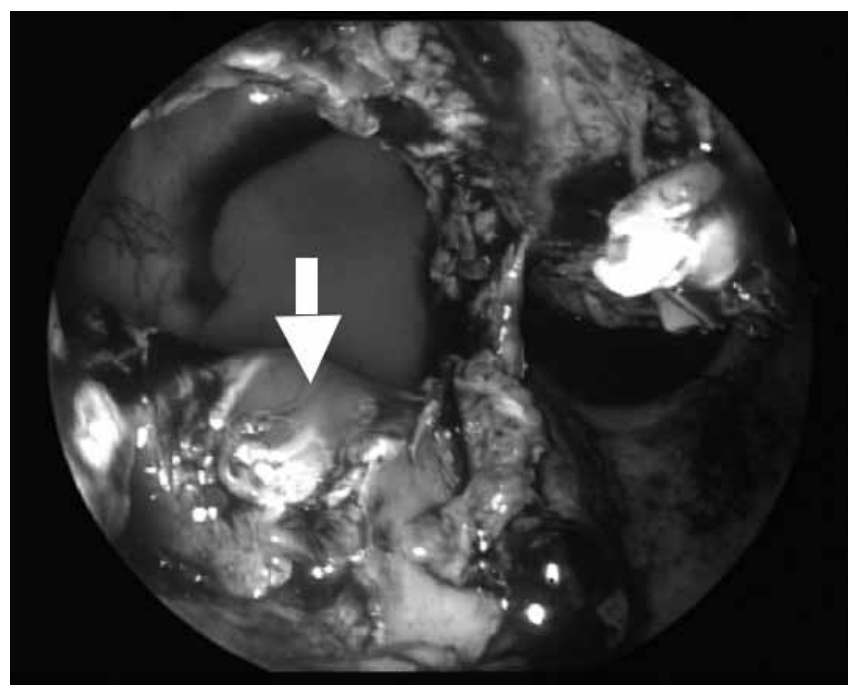

Figure 3. Endoscopic photograph showing the radicular cyst in the right maxillary sinus. At the opening of radicular cyst, a purulent discharge was noted (arrow).

the endoscopic sinus surgery for the treatment of chronic maxillary sinusitis of dental origin after the dental treatment of odontogenic lesions. Cedin et al. ${ }^{\left({ }^{8}\right)}$ first removed the cyst walls from the maxillary sinus using small clamps with an endoscope through a $10 \mathrm{~mm}$ circumferential hole of the canine fossa. Recently, transnasal removal of large dentigerous cysts of the maxillary sinus has been also reported using the endoscopic approach through the middle meatus ${ }^{(11,12)}$.

In the present study, a transnasal endoscopic approach was used to remove the cyst walls of odonotogenic cysts through the maxillary sinus and the opening of inferior meatus. This is the first report to remove the cyst walls of the radicular cyst with no incision of oral mucosa. This technique can be applied in cases with odontogenic maxillary cysts. It is less invasive than conventional dental procedures, and it prevents oroantral fistula formation and chronic maxillary sinusitis. There were no complications during and after the surgery in this series.

We removed the cyst walls completely from the maxillary sinus in five of ten patients with a radicular cyst. Another five patients undwernt the partial resection of the cyst walls of the radicular cyst. However, no recurrence has been noted after 11 to 72 months of follow-up. These results indicate that the partial resection of the cyst wall may be sufficient for the endoscopic treatment of the radicular cysts. In patients with a dentigerous cyst, we completely removed the cyst walls in all three cases. Because malignant change in the dentigerous walls cysts have been reported ${ }^{(10,13)}$, the walls of the dentigerous cysts should be completely removed.

Teeth of the damaged affected radicular cyst should be extracted, as inadequate dental treatment may cause the recurrence of the cyst ${ }^{(14)}$. Most of the affected teeth have usually been extracted by the traditional dental procedure. In the present 

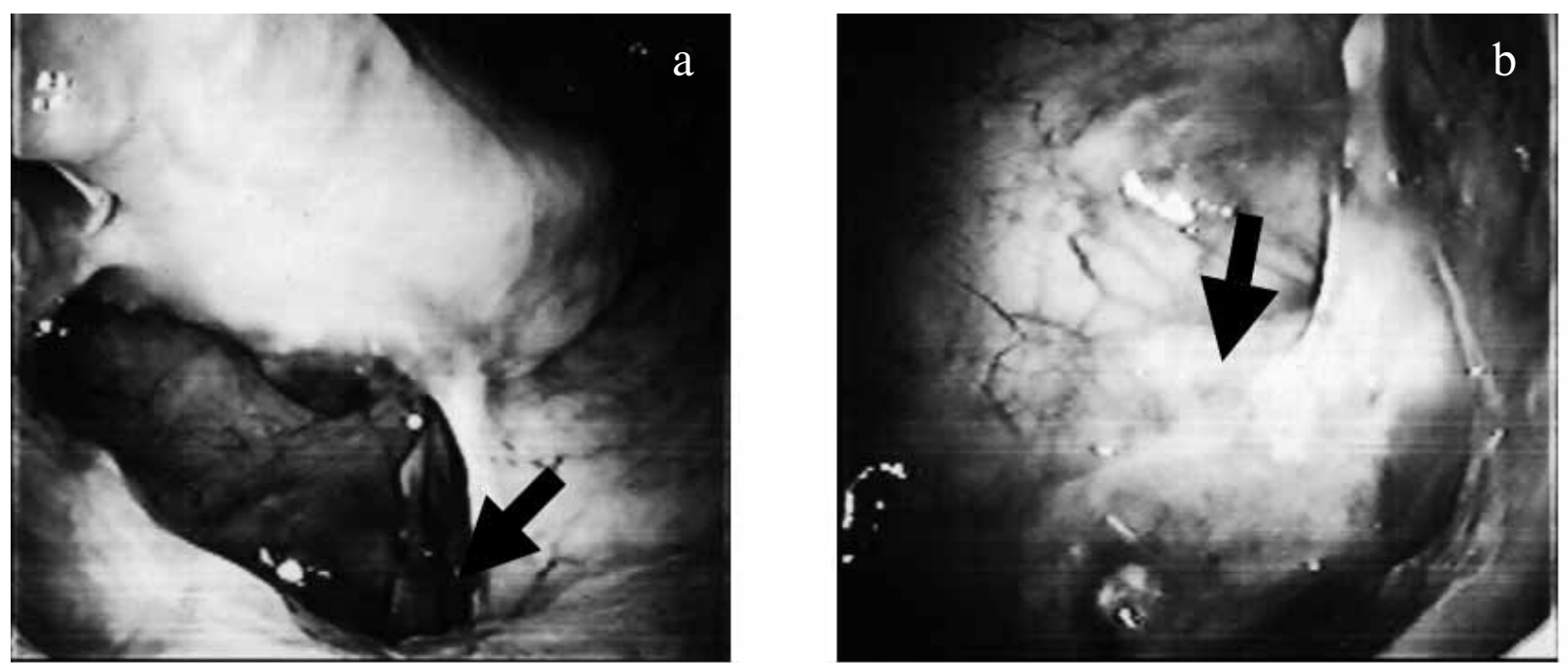

Figure 5. Endoscopic photograph of the inferior meatus (a) and right maxillary sinus (b) at 3 months after the surgery. The floor of the maxillary sinus was well epithelialized (arrows).
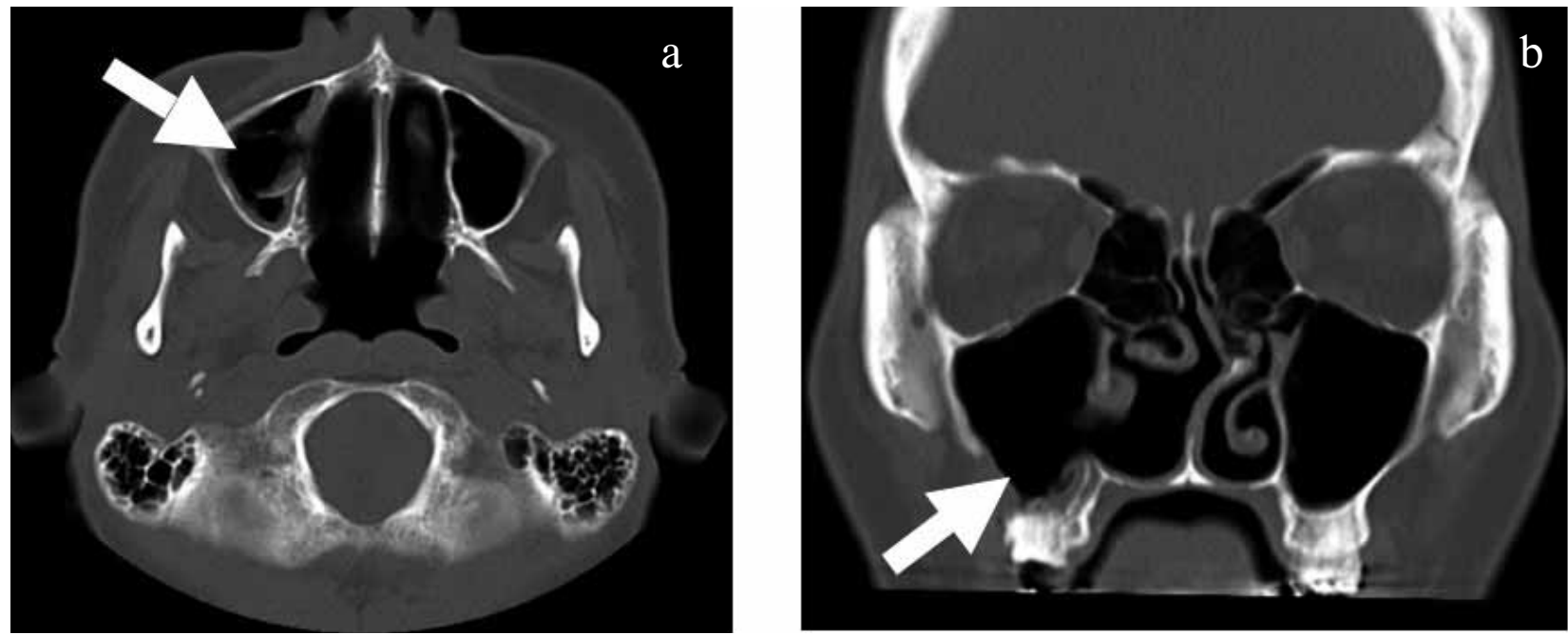

Figure 6. Axial (a) and coronal (b) CT scan at 6 months after the surgery. The complete removal of the radicular cyst was confirmed (arrows).

study, we could preserve the undamaged affected teeth in seven of ten patients with a radicular cyst. Although the dental treatment of the affected teeth is important prior to the endoscopic procedure, this approach can preserve the affected teeth in cases of radicular cysts. We also preserved the affected tooth in one of three patients with a dentigerous cyst, in the condiotn that the tooth was expected to grow in the correct position.

\section{REFERENCES}

1. Jameson MJ, Kountakis SE. Endoscopic management of extensive inverted papilloma. Am J Rhinol 2005; 19: 446-451.

2. Hofmann T, Bernal-Sprekelsen M, Koele W, Reittner P, Klein E, Stammberger H. Endoscopic resection of juvenile angiofibromaslong term results. Rhinology 2005; 43: 282-289.

3. Silva LR, Santos RP, Zymberg ST. Endoscopic endonasal approach for cerebrospinal fluid fistulae. Minim Invasive Neurosurg 2006; 49: 88-92.

4. Hinohira Y, Yumoto E, Shimamura I. Endoscopic endonasal reduction of blowout fractures of the orbital floor. Otolaryngology Head Neck Surg 2005; 133: 741-747.
5. Suzuki M, Sakurai H, Seno S, Kitanishi T, Shimizu T. Use of realtime magnetic resonance image guidance in endoscopic sinus surgery. Minim Invasive Ther Allied Technol 2005; 14: 376-384.

6. Seno S, Arikata M, Sakurai H, Owaki S, Fukui J, Suzuki M, and Shimizu T. Endoscopic ligation of the sphenopalatine artery and the maxillary artery for the treatment of intractable posterior epistaxis. Am J Rhinol, in press.

7. Daley TD, Wisoccki GP, Pringle GA. Relative incidence of odontogenic tumors and oral jaw cysts in Canadian population. Oral Surg 1994; 77: 276-280.

8. Cedin AC, Paula Junior FA, Landim ER, Silva FL, Oliveira LF, Sotter AC. Endoscopic treatment of odontogenic cyst with intrasinusal extension. Rev Bras Otorrinolaringol (Engl Ed) 2005; 71: 392-395.

9. Suarez-Conqueiro MM, Schoen R, Schramm A, Gellrich NC, Schmelzeisen R. Endoscopic approach to removal of an ectopic mandibular third molar. Br J Oral Maxillofac Surg 2003; 41: 340342.

10. Costa F, Emanuelli E, Robiony M, Zerman N, Polini F, Politi M. Endoscopic surgical treatment of chronic maxillary sinusitis of dental origin. J Oral Maxillofac Surg 2007; 65: 223-228.

11. Bradfield WJ, Broadway ES. Malignant change in a dentigerous cyst. Br J Surg 1958; 45: 657-659. 
12. Di Pasquale P and Shermetaro C. Endoscopic removal of a dentigerous cyst producing unilateral maxillary sinus opacification on computed tomography. Ear Nose Throat J 2006; 85: 747-748.

13. Micozkadioglu SD, Erkan AN. Endoscopic removal of a maxillary dentigerous cyst. B-ENT. 2007; 3: 213-216.

14. Gulbranson SH, Wolfrey JD, Raines JM, McNally BP. Squamous cell carcinoma arising in a dentigerous cyst in a 16-month-old girl. Otolaryngol Head Neck Surg 2002; 127: 463-464.

15. Kulacz R, Fishman G, Levine H. An unsuccessful sinus surgery caused by dental involvement within the floor of the maxillary sinus. Oper Tech Otolaryngol - Head Neck Surg 2004; 15: 2-3.
Satoshi Seno, M.D.

Department of Otorhinolaryngology

Shiga University of Medical Science

Tsukinowa Seta

Otsu

Shiga 520-2143

Japan

Tel: $+81-77-548-2264$

Fax: 81-77-548-2783

E-mail: senosato@belle.shiga-med.ac.jp

\section{International}

\section{MASTERCOURSE ON ENDOSCOPIC SINUS SURGERY} 5-6-7-8 May 2010 - Brussels, Belgium

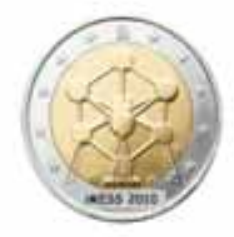

International faculty: P. CLEMENT (Brussels), L. CLOSE (New York), O. MICHEL (Brussels), S. SCHAEFER (New York), W. THUMFART (Innsbruck), J. ZINREICH (Baltimore) Specials guests: G. BACHMANN-HARILDSTAD (Oslo), J. D'HAENS (Brussels), E. PASQUINI (Bologna), WANG D.Y. (Singapore) Anatomy: J. CLARYS Guest lectures: S. Halewyck, Van Rompaey K.

\section{All in English}

Lectures, video sessions, live surgery, cadaver head demonstration, extensive hands-on cadaver dissection with CT from each head, intensive interactive discussion with the faculty members.

Advanced techniques: HRCT/MR, fusing, erasing, navigation, frontal sinus, anterior skull base, CSF-leakage, DCR, sphenoid, benign and malign paranasal sinus tumors, pituary gland surgery, pterygo-palatine fossa and beyond...

Registration fee ENT specialist $1450 €$, ENT resident in training (certificate requested) $1250 €$, Lectures only $1100 €$, Accompanying person $450 €$ Registrations/info/course secretariat

UZ BRUSSEL (Hospital Vrije Universiteit Brussels) Dept. of ENT, H\&N Surgery, Prof. Dr. O. Michel c/o Mrs. K. Nuyts, Laarbeeklaan 101, B-1090 Brussels, Belgium, karine.nuyts@uzbrussel.be or fax+32/2-4776880 